\title{
Propuesta de un modelo pedagógico e-learnig en la universidad de san martín de porres
}

\section{Proposal for an e-learning pedagogical model at the university of san martín de porres}

\author{
DOI: $10.46932 / \mathrm{sfjdv2n4-062}$
}

Received in: May 1st, 2021

Accepted in: Jun 30th, 2021

\section{Dra. Milagros Cecilia Huamán Castro}

Formación académica superior: PhD en Estrategias Digital E-learning - Doctora en Educación Institución actual: Universidad de San Martín de Porres

Domicilio completo: Av. Sergio Bernales 599. Surquillo, Lima, Perú.

Correo electrónico: milagros.huaman.castro@gmail.com

\section{RESUMEN}

El artículo tiene como objetivo dar a conocer el modelo pedagógico en el cual se desarrollan los cursos virtuales de pregrado y posgrado de la Universidad de San Martín de Porres, el cual se viene aplicando con éxito desde enero del 2014 en todos los programas virtuales, este incluye una Metodología virtual propia y un Diseño Pedagógico ágil. El modelo pedagógico virtual implica desde el proceso de cuatro meses previos a la creación del curso con el uso de diversos documentos que norman el proceso, la capacitación a los docentes y alumno, y la aplicación de rúbricas y matrices de revisión según estándares internacionales que garantizan la calidad de los programas virtuales en la comunidad internacional, hasta el desarrollo de la metodología virtual durante el proceso de enseñanza aprendizaje en línea en un aula virtual organizada en cuatro dimensiones.

Palabras clave: modelo pedagógico, metodología virtual, conectivismo, curso virtual, calidad.

\section{ABSTRACT}

The article aims to present the pedagogical model in which the undergraduate and graduate virtual courses of the Universidad de San Martín de Porres are developed, which has been successfully applied since January 2014 in all virtual programs, this includes its own virtual methodology and an agile pedagogical design. The virtual pedagogical model involves from the process of four months prior to the creation of the course with the use of various documents that regulate the process, the training of teachers and students, and the application of rubrics and review matrices according to international standards that guarantee the quality of virtual programs in the international community, to the development of the virtual methodology during the online teaching-learning process in a virtual classroom organized in four dimensions.

Key words: pedagogical model, virtual methodology, connectivism, virtual course, quality.

\section{INTRODUCCIÓN}

La necesidad del siglo XXI que la educación se brinde en modalidad virtual rompiendo las barreras del tiempo y el espacio, conlleva a la creación de programas virtuales que cumplan los 
estándares de calidad que ofrecemos en nuestros programas en modalidad presencial, por ello la Universidad San Martín de Porres, previas investigaciones de experiencias y buenas prácticas internacionales, ha diseñado un modelo pedagógico para cursos virtuales considerando dos pilares; la metodología de cursos virtuales por créditos y horas académicas, y el diseño pedagógico del aula virtual.

Partimos del fundamento pedagógico de dos corrientes contemporáneas que lo respaldan, que son el constructivismo y el conectivismo, las cuales nos llevan al logro de un aprendizaje significativo en el entorno virtual. Con esta base hemos podido planificar, organizar y diseñar nuestros programas virtuales, que responden a una evaluación por competencias, basados en estándares internacionales de calidad.

Nuestro modelo pedagógico cuenta con una metodología para sesiones de clase virtual por créditos y horas académicas, la cual se da en dos fases; diseño de cursos o programas virtuales, que cuenta con tres etapas, la primera consiste en la elaboración y desarrollo de un cronograma de Virtualización (arranques de cursos), la elaboración en 12 semanas de la estructura del curso que consta de cuatro módulos en un mes y la certificación de calidad del curso a través de dos niveles de revisión y validación, primero calidad de la USMP y luego, calidad de Academic PartnerShips bajo los estándares de calidad de Quality Matters (QM) y Quality Scorecard (QSC), rúbricas diseñadas para validar programas virtuales. La segunda fase consiste en el desarrollo de la sesión de clase virtual por crédito hora, que se da por módulos de una semana. Allí cada semana considera 4 días (lunes a jueves) el desarrollo de las diversas actividades, el viernes está destinado a completar alguna actividad pendiente y la metacognición, mientras que los sábados se programa una retroalimentación semanal y completar la metacognición quienes faltan, finalmente, los domingos se procede a la evaluación y publicación de notas por parte del docente. Todo ello enmarcado en la propuesta de un crédito por hora académica de 45 minutos.

Finalmente, nuestro modelo pedagógico se complementa con un diseño pedagógico del aula virtual, estructurado en cuatro dimensiones; informativa, formativa, experiencial y comunicativa. Interfaz gráfica que se utiliza en las plataformas donde desarrollamos nuestros programas virtuales con éxito y buen acogida.

\section{FUNDAMENTOS DEL MODELO PEDAGÓGICO E-LEARNING}

La necesidad de toda institución educativa es contar con un Modelo Pedagógico, que sea la base del desarrollo de las asignaturas, para el logro de los objetivos trazados. Sin este, el proceso educativo 
carecería de consistencia, calidad y sustento. Para ello, es importante abordar las principales corrientes pedagógicas contemporáneas que lo respalden.

\subsection{EL CONSTRUCTIVISMO}

Es una posición compartida por diferentes tendencias de la investigación psicológica y educativa. Entre ellas se encuentran las teorías de Jean Piaget (1952), Lev Vygotsky (1978), David Ausubel (1963), Jerome Bruner (1960), y aún cuando ninguno de ellos se denominó como constructivista sus ideas y propuestas claramente ilustran las ideas de esta corriente.

El Constructivismo, dice Méndez (2002) “es en primer lugar una epistemología, es decir una teoría que intenta explicar cuál es la naturaleza del conocimiento humano". El constructivismo asume que nada viene de nada. Es decir que el conocimiento previo da nacimiento a conocimiento nuevo.

El constructivismo sostiene que el aprendizaje es esencialmente activo. Una persona que aprende algo nuevo, lo incorpora a sus experiencias previas y a sus propias estructuras mentales. Cada nueva información es asimilada y depositada en una red de conocimientos y experiencias que existen previamente en el sujeto, como resultado podemos decir que el aprendizaje no es ni pasivo ni objetivo, por el contrario es un proceso subjetivo que cada persona va modificando constantemente a la luz de sus experiencias. Abbott (1999).

El constructivismo constituye todo un marco explicativo en el que confluyen teorías psicológicas y pedagógicas que abordan la actividad del estudiante como un proceso de adquisición del conocimiento en forma dinámica y total, ya sean los saberes especializados como las matemáticas, u otros saberes como el conocimiento de los estilos de vida, como producto de las representaciones mentales que construyen los educandos en la experiencia misma. Vargas (2006).

Los antecedentes del paradigma constructivista se encuentran fuertemente cimentados en los trabajos de Lev S. Vigotsky y de Jean Piaget, y tiene un marcado énfasis en una búsqueda epistemológica sobre cómo se conoce la realidad, cómo se aprende, en otras palabras, la génesis y desarrollo del conocimiento y la cultura.

El constructivismo plantea el pleno y consciente desarrollo del pensamiento y el lenguaje mediante actividades en las que el maestro "enseña”, sólo hasta después que los educandos han intentado por sus propios medios y con la ayuda de él y de otros alumnos del grupo, a desarrollar las actividades, lo cual mueve a cada alumno en su "zona de desarrollo próximo" de modo tal que pasen de un estado de "no saber" a "saber" y de otro de "no saber hacer" a uno de "saber hacer".

Para lograr la construcción del conocimiento el maestro debe planear "situaciones de aprendizaje grupal colaborativo" en las que además de tener en cuenta qué se aprende, se tiene muy en consideración 
el cómo, dónde, cuándo, de manera tal de propiciar e intensificar las relaciones interpersonales de cada sujeto y del grupo en un contexto social determinado.

Mediante la creación de situaciones de aprendizaje grupal colaborativo el maestro desarrolla una enseñanza indirecta donde el énfasis está en la actividad y comunicación con momentos de reflexión, de búsqueda y procesamiento de la información, así como de comunicación creativa de los procesos y resultados, todo lo cual desarrolla las potencialidades y la autonomía del que aprende.

En cuanto al conocimiento, el constructivismo plantea que su valor no es absoluto, pues éste es el producto de las múltiples interpretaciones que hacen los individuos de su entorno, de acuerdo a las posibilidades de cada uno para interactuar y reflexionar. Los sujetos negocian significados a partir de la observación y valoración de aspectos de la realidad que les son comunes. Los alumnos desarrollan sus propias estrategias de aprendizaje, señalan sus objetivos y metas, al mismo tiempo que se responsabilizan de qué y cómo aprender. La función del profesor es apoyar las decisiones del alumno (Gros, 1997, p. 99).

\subsection{EL CONECTIVISMO}

Siguiendo con los enfoques, George Siemens en su "Teoría de aprendizaje para la era digital" (2004) indica que un principio central de la mayoría de las teorías del aprendizaje, es que el aprendizaje ocurre dentro de una persona. Incluso los enfoques del constructivismo social, los cuales sostienen que el aprendizaje es un proceso social, promueven el protagonismo del individuo en el aprendizaje. Estas teorías no hacen referencia al aprendizaje que ocurre por fuera de las personas (el aprendizaje que es almacenado y manipulado por la tecnología). Siemens nos indica que, también fallan al describir cómo ocurre el aprendizaje al interior de las organizaciones.

Sintetizando, el conectivismo, según la teoría de Siemens, es la integración de principios explorados por las teorías de caos, redes, complejidad y auto-organización. El aprendizaje es un proceso que ocurre al interior de ambientes difusos de elementos centrales cambiantes - que no están por completo bajo control del individuo. El aprendizaje (definido como conocimiento aplicable) puede residir fuera de nosotros (al interior de una organización o una base de datos), está enfocado en conectar conjuntos de información especializada, y las conexiones que nos permiten aprender más, tienen mayor importancia que nuestro estado actual de conocimiento. Por ello, el conectivismo es orientado por la comprensión que las decisiones están basadas en principios que cambian rápidamente. Continuamente se está adquiriendo nueva información. La habilidad de realizar distinciones entre la información importante y no importante resulta vital. También es crítica la habilidad de reconocer cuándo una nueva información altera un entorno basado en las decisiones tomadas anteriormente. 
Es importante resaltar que el punto de partida del conectivismo es el individuo. El conocimiento personal se compone de una red, la cual alimenta a organizaciones e instituciones, las que a su vez retroalimentan a la red, proveyendo nuevo aprendizaje para los individuos. Este ciclo de desarrollo del conocimiento le permite a los aprendices estar actualizados en su área mediante las conexiones que han formado. Es así que el Cmap Tools es la herramienta idónea para desarrollar este conocimiento, ya se va formando una red de ideas, constructos a lo largo de su creación, que se da a través de la web en un entorno virtual, en el cual todos los participantes están conectados, procesando ideas para un mismo fin.

Si realizamos una comparación, podríamos decir que, la tubería es más importante que su contenido. Así, nuestra habilidad para aprender lo que necesitamos mañana es más importante que lo que sabemos hoy. Un verdadero reto para cualquier teoría de aprendizaje es activar el conocimiento adquirido en el sitio de aplicación. Sin embargo, cuando el conocimiento se necesita, pero no es conocido, la habilidad de conectarse con fuentes que corresponden a lo que se requiere es una habilidad vital. A medida que el conocimiento crece y evoluciona, el acceso a lo que se necesita es más importante que lo que el aprendiz posee actualmente.

El conectivismo presenta un modelo de aprendizaje que reconoce los movimientos tectónicos en una sociedad en donde el aprendizaje ha dejado de ser una actividad interna e individual. La forma en la cual trabajan y funcionan las personas se altera cuando se usan nuevas herramientas. El área de la educación ha sido lenta para reconocer el impacto de nuevas herramientas de aprendizaje y los cambios ambientales, en la concepción misma de lo que significa aprender. El conectivismo provee una mirada a las habilidades de aprendizaje y las tareas necesarias para que los aprendices florezcan en una era digital.

\subsection{APRENDIZAJE SIGNIFICATIVO}

Es importante mencionar, que mediante la realización de aprendizajes significativos, el alumno construye, modifica, diversifica y coordina sus esquemas, estableciendo de este modo redes de significados que enriquecen su conocimiento del mundo físico, social y potencian su crecimiento personal. Coll (1991).

Según Sánchez et al., (2008, 2009 y 2011), este proceso de mejora debe ser interactivo y se debe sustentar en los siguientes principios: a) mayor implicación y autonomía del estudiante; b) utilización de metodologías activas de trabajo en equipo, tutorías, etc.; c) el docente debe ser un agente creador de escenarios u entornos de aprendizaje contextualizados que estimulen a los alumnos.

Aprendizaje significativo es aprendizaje con comprensión, con significado, con capacidad de transferencia. Es el opuesto del aprendizaje mecánico. Si imaginamos que el aprendizaje se produce a lo 
largo de un continuo, aprendizaje mecánico estaría en un extremo y aprendizaje significativo en el otro. Moreira (2012).

Es así que podemos definir el aprendizaje significativo como un proceso interactivo que brinda autonomía al estudiante, ya que construye y modifica esquemas para potenciar su crecimiento personal en un contexto determinado, donde trabaja en equipo con metodologías activas y una buena tutorización.

Este es el logro de nuestro modelo pedagógico, que promueve la interacción en el aula virtual, con un tutor atento a dinamizar las comunicaciones, promoviendo el trabajo colaborativo a través de herramientas virtuales. Todo ello enmarcado en la retroalimentación permanente que le permite potenciar su crecimiento personal y social.

\section{METODOLOGÍA PARA SESIONES DE CLASE}

Nuestra metodología para desarrollar sesiones de clase virtual responde a la necesidad de poder demostrar en el entorno académico, que nuestros cursos virtuales cuentan con estándares de calidad y permiten desarrollar las mismas competencias y habilidades que los presenciales, de acuerdo a la cantidad de créditos que tiene cada uno, sin que exista una diferencia que le reste competitividad en el entorno académico a los alumnos graduados. Esta metodología se desarrolla en dos fases:

\section{Fase 1: Diseño de cursos}

El proceso de planificación y diseño de la instrucción de un curso para entornos virtuales de aprendizaje, es fundamental y debe considerar de manera anticipada y teniendo en cuenta los grupos a formar y los propósitos de formación a lograr, las diferentes estrategias para que el estudiante aprenda interactuando con las variadas actividades, con sus compañeros, con los contenidos, con sus tutores, ya sea de manera individual o colaborativa, buscando los aprendizajes significativos, prácticos y creativos. Jurado (2010).

El diseño de cursos se desarrolla a lo largo de un proceso que implica el cumplimiento de tres etapas:

\section{Cronograma proceso de virtualización}

Es el primer documento que recibe el docente del curso virtual, para iniciar su proceso de elaboración de materiales. Este cuenta con una duración de 12 semanas, después de este tiempo, deberá tener el curso listo en la plataforma, para pasar a su revisión.

La primera actividad registrada que debe cumplir es una capacitación virtual para conocer la plataforma donde habilitará su curso, los estándares de calidad que requiere para crear materiales y 
conocer el proceso de virtualización. Luego, de la semana 1 a la 6 deberá elaborar la Estructura del curso y organizar sus cuatro módulos.

Culminado, enviará la Estructura del curso al equipo de Academic PartnerShips en Estados Unidos, para que procedan a revisarla. La semana 7 y 8 iniciará su Autocapacitación y la implementación del curso en la plataforma Canvas.

Estando ya el curso listo en el aula, la semana 9 y 10 previa revisión de calidad de la USMP, pasará a revisión de AP bajo estándares Internacionales de calidad. Paralelamente la USMP procedemos a la capacitación virtual del docente que asumirá la tutoría del curso (no siempre quien lo crea es quien lo tutoriza). Finalmente, la semana 11 y 12 el docente que elaboró el curso, deberá levantar las observaciones que envíe AP y dejarlo listo, para su inicio.

Figura 1: Modelo de cronograma de virtualización

\begin{tabular}{|c|c|c|c|}
\hline \multicolumn{4}{|c|}{$\begin{array}{l}\text { CRONOGRAMA PROCESO DE VIRTUALIZACIÓN } \\
\text { DE UN CURSO VIRTUAL } \\
\text { (ARRANQUE 10) }\end{array}$} \\
\hline Inicio & DURACION & ACTMDAD & RESPON SABLE \\
\hline $\begin{array}{c}\text { Mares } \\
\text { Mas-02-14 }\end{array}$ & 1e.da & 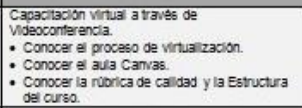 & $\begin{array}{l}\text { cagagasos } \\
\text { cusup }\end{array}$ \\
\hline $\begin{array}{c}\text { Marts } \\
05-08-14\end{array}$ & Senaras 13.3 & 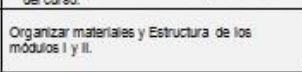 & 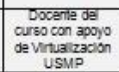 \\
\hline$\underset{25-085-14}{\text { Lunes }}$ & Senana 436 & $\begin{array}{l}\text { Organizar materases y Estuctura de los } \\
\text { mosulos III YN. }\end{array}$ & $\begin{array}{l}\text { Docente dal } \\
\text { ourso con appyo } \\
\text { de Vinualzzalon } \\
\text { USMP }\end{array}$ \\
\hline $\begin{array}{l}\text { Domingo } \\
14-09-14\end{array}$ & 10 la & 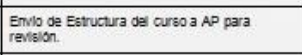 & Persona AP \\
\hline $\begin{array}{l}\text { Lunes } \\
15-09-14\end{array}$ & semana $7 y$ s & 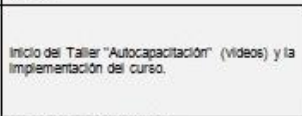 & 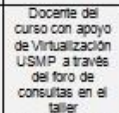 \\
\hline $\begin{array}{ll}\text { Lunes } \\
20-0914\end{array}$ & Senana 9 y 10 & 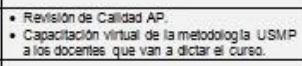 & 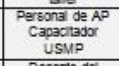 \\
\hline$\underset{13-102-14}{\text { Lunes }}$ & semana 11 y 12 & Correcconess aelmingasas. & 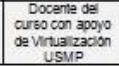 \\
\hline
\end{tabular}

-e corsoinclas Iunes 27 da octubra de 2014

\section{Estructura general del curso}

Documento muy laborioso y base indispensable para la creación del curso, donde se detallan los datos generales del docente y la información que se va a desarrollar en cada uno de los módulos como; la descripción, objetivos, video de presentación, separatas, ppt, lecturas, videos, enlaces, tareas, exámenes, prácticas, actividades no calificadas, foros, chat, videoconferencias. Así como la bibliografía, glosario y fórmula de evaluación del curso.

El tiempo estimado para su elaboración es de 6 semanas, lo cual va paralelamente con la tarea de organizar los materiales para cada módulo. La elaboración de esta estructura requiere que el docente 
experto haya previamente investigado y recopilado información en su portafolio digital, para crear sus materiales acorde a la necesidad del curso.

La estructura se presenta en 4 módulos, cada semana se desarrolla uno. Cada actividad definida en la estructura incluye el tiempo que tomará al estudiante desarrollarla, lo cual conlleva a que las actividades se desarrollen en sesiones, cuya duración dependerá del número de créditos o de las horas académicas del curso. No será la misma cantidad de actividades que programe para un curso de un crédito que para uno de cuatro.

\section{Certificación de calidad del curso}

Los cursos virtuales solo quedan listos y aprobados para habilitarse al público, cuando Calidad de la USMP los ha revisado y AP bajo sus estándares de calidad para cursos virtuales Quality Matters y Qualitiy Scorecard los ha aprobado. Para esta revisión de calidad, AP cuenta con una matriz de evaluación formada por 43 indicadores distribuidos en 9 criterios, la cual se denomina Rúbrica de Revisión de Calidad de Curso de Academic Partnerships. Esta a su vez contiene indicadores basados en Academic Partnerships effective practices.

Figura 2. Matriz de revisión de calidad de cursos virtuales

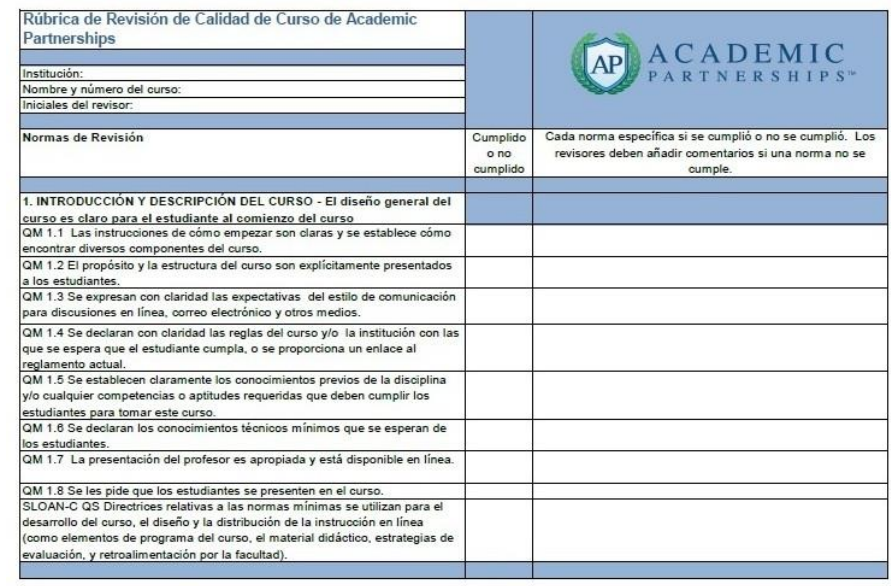

De no cumplir los cursos con los estándares de calidad que se han definido, no pueden habilitar al público, primero deben pasar por un proceso de corrección. Por este motivo, se requiere que los cursos estén listos en el aula virtual un mes antes de su inicio, ya que la revisión de AP demora 15 días promedio y levantar las observaciones, 5 días. Quedando el curso listo 10 días antes de su inicio.

El docente que elabora el curso es el responsable hasta dejarlo listo, una vez finalizada su labor, el virtualizador asignado que lo acompañó hasta ese momento, procede a completar una Ficha de entrega de curso, firmada por él y la jefatura de Tecnología Educativa de la USMP. Recién procede a gestionarse su pago, por el servicio brindado. Finalizando así el proceso de elaboración /diseño del curso virtual. 
El diseño de los cursos virtuales implica un trabajo muy laborioso, pero sobretodo debe ser de calidad, por ese motivo nuestros cursos pasan por unos indicadores de revisión de calidad que los respaldan internacionalmente. Surge la pregunta ¿cómo pueden los diseñadores de cualquier curso en línea asegurarse de que están incorporando indicadores y estándares de calidad adecuados en sus cursos? ¿Y quién puede ayudarlos en ese esfuerzo? Para ello, se crearon los Quality Matters ® y Quality Scorecard ®. Estas dos herramientas ofrecen rubros y categorías fáciles de usar que los diseñadores de instrucción y administradores pueden optar por aplicar en la creación y evaluación de sus cursos y programas.

Si bien hay una serie de rúbricas utilizadas para evaluar los programas de aprendizaje en línea, los más utilizados por las instituciones de educación superior incluyen Quality Scorecard de Sloan-C para la administración de los programas de educación en línea, y la rúbrica Quality Matters.

Quality Matters (QM) Rúbrica compuesta por 8 estándares generales y 40 criterios usados para evaluar el diseño de cursos virtuales y semipresenciales. Fue desarrollada para el periodo 2011-2013. Es un programa desarrollado por el Departamento of Educación de los Estados Unidos de Norteamérica (The Quality Matters Rubric, 2008) cuyo resultado ha sido el desarrollo de un modelo de mejora continua para evaluar y garantizar la calidad de los cursos en línea.

La rúbrica Quality Matters (QM) es un proceso de revisión por pares que está diseñado para certificar la calidad de los cursos en línea y mixtos. El 2008-2010 Quality Matters Rúbrica es la edición más actual que abarca el diseño en el curso online / híbrido. El enfoque de este rubro se debe principalmente a la calidad en el nivel del curso. Una particularidad de la rúbrica Quality Matters es el concepto de "alineación". Este ocurre cuando los componentes fundamentales de un curso trabajan juntos garantizando que los estudiantes alcancen los objetivos de aprendizaje previstos. Romero (2011).

Quality Scorecard (QSC) Este cuadro de mando o matriz se centra en la evaluación de la calidad de los programas en línea. Permite la medición y cuantificación de elementos de calidad dentro de los programas en línea en la educación superior.

Mediante la evaluación de cada uno de los indicadores de calidad respectivos dentro de las nueve categorías establecidas, se puede determinar las fortalezas y debilidades de un programa virtual. Los resultados de esta herramienta se pueden utilizar para apoyar la mejora del programa y las iniciativas de planificación estratégica. La rúbrica Qualitiy Scorecard también se puede utilizar para demostrar a los organismos de acreditación, los elementos de la calidad dentro del programa, así como un nivel general de calidad. 


\section{Fase 2: Desarrollo de la sesión de aprendizaje por crédito hora}

La metodología para sesiones de clase virtual se basa en los fundamentos del Constructivismo, en el cual el docente no es el centro del proceso de aprendizaje, ni tampoco lo es el contenido como en el Conductivismo. No existe el dictado ni trasferencia de conocimientos, sino el acompañamiento del tutor /docente, para lograr un aprendizaje significativo a lo largo del proceso.

¿Por qué aprender en un contexto de aprendizaje constructivista virtual?

El constructivismo presenta escenarios de aprendizaje diversificados y hace uso de todos los medios y recursos posibles para aprender (recursos de la Web 2.0).

$\checkmark \quad$ Permite la interactuación con los objetos del conocimiento y con otras personas que poseen conocimientos (docentes, tutores, compañeros) En foros, chat, videoconferencias, etc.

$\checkmark \quad$ Pone énfasis en el entorno cultural y natural de los participantes, comprendiendo que las realidades de los mismos son diversas. Promueve la atención personalizada de los estudiantes según sus estilos y ritmos de aprendizaje. Inteligencias múltiples" (actividades contextualizadas).

$\checkmark \quad$ El error es un elemento importante, pues, promueve que el estudiante tome conciencia y resuelva una situación problemática. Es retador y permite la retroalimentación (retroalimentación constante y al finalizar cada módulo).

$\checkmark \quad$ Propicia el trabajo colaborativo y cooperativo. Se promueve la práctica de valores como la solidaridad, tolerancia, respeto mutuo y equidad (uso de wikis, cmap tools, redes, etc).

$\checkmark \quad$ El contenido educativo se desarrolla en tres dimensiones: Conceptual (saber saber), procedimental (saber hacer) y actitudinal (saber ser). Evaluación por competencias.

$\checkmark \quad$ El docente se convierte en actor mediador entre el estudiante y el objeto de conocimiento, propone situaciones de aprendizaje que generen retos cognitivos (facilitador). Busca que los contenidos educativos sean asimilables por los estudiantes (lo acompaña en el proceso diario de enseñanza-aprendizaje).

Tanto el docente como el alumno cumplen diferentes funciones en la educación virtual, pero, finalmente, tienen un punto de intersección, el aprendizaje significativo. Es así que es indispensable que el docente virtual conozca las herramientas tecnológicas de la Web 2.0 (Moodle LMS, Canvas LMS, Chamilo LMS; Redes sociales; Servicio Micro Blogging, etc.). Y, por otro lado, el alumno virtual sea constructor de su propio conocimiento a través de la utilización de esas herramientas. Es por ello que se exige que el docente adecúe, modifique y reestructure los procesos, la didáctica, la metodología de la enseñanza-aprendizaje de la modalidad presencial a la enseñanza - aprendizaje en la modalidad virtual, pues es imposible trabajar con los mismos criterios en la educación virtual. 
"Por otra parte, un error que siempre hemos cometido con las nuevas tecnologías, y que ha llevado a que las mismas no desarrollen todas las posibilidades que presentan para la creación de nuevos entornos formativos, es el deseo de trasladar sobre ellas principios aplicados, de la enseñanza presencial o de tecnologías más tradicionales" (Cabero, 2006).

La metodología también se basa en Academic Partnerships effective practices, y en los estándares de calidad para cursos virtuales: Quality Matters y Qualitiy Scorecard, en los cuales, la cantidad de tiempo destinado al estudio de un adulto que trabaja dentro de un ambiente virtual debe ser entre 15 a 20 horas semanales, considerando además el formato acelerado que tiene el curso.

Debemos tener en cuenta que el perfil del estudiante es una persona adulta que muchas veces trabaja o desarrolla durante el día otro tipo de actividades, por eso elige la modalidad virtual, por ser más flexible, y desarrollarlo por las noches o sus momentos libres. Además, también debemos considerar a los que tienen carga familiar y los que tienen capacidades diferentes (estilos de aprendizaje diferenciados).

Se consideró trabajar la metacognición ya que esta implica reflexionar sobre nuestro propio pensamiento o conocimiento sobre nuestros aprendizajes, así como sobre nosotros mismos en cuanto a aprendices. Klenowski (2005). Después de estudiar distintas definiciones y averiguaciones empíricas de la metacognición, Hacker (1998) concluye que es importante que los seres humanos se comprendan así mismos como agentes de su propio pensamiento nuestro pensamiento puede ser controlado y regulado de forma deliberada". Lo cual es muy importante en el desarrollo académico en el entorno virtual.

Además, adicional a la retroalimentación permanente durante todo el proceso académico de la semana, se ha programado en las sesiones virtuales una retroalimentación grupal al finalizar cada módulo, así el estudiante puede compartir sus inquietudes con los demás compañeros, con el acompañamiento del tutor virtual, y las respuestas del experto serán de utilidad a todos los alumnos.

El concepto de retroalimentación es cibernético y fue tomado por Bertalanffy en su empeño por explicar la manera como los sistemas se autorregulan. La idea central de la cibernética es la retroalimentación definida así por Wiener. La retroalimentación es un método para controlar un sistema reintroduciéndole los resultados se su desempeño en el pasado. Si estos resultados son utilizados meramente como datos para evaluar el sistema y su regulación, tenemos la retroalimentación simple; pero si esa información de retorno sobre el desempeño anterior del sistema puede modificar el proceso general y su pauta de desempeño actual, tenemos un proceso que puede llamarse aprendizaje. Gallegos. (2006). Lo cual en nuestro sistema académico virtual es de mucha utilidad al docente, para la mejora continua y logro del aprendizaje significativo, que es nuestra meta.

A continuación las tablas de cantidad de créditos que utilizamos con su equivalente a las horas académicas que hemos definido en el entorno virtual. Resaltamos que se ha definido trabajar de lunes a 
jueves todas las actividades como; tareas individuales y grupales, foros temáticos y de debate, quedando el viernes para que los alumnos puedan completar las tareas pendientes (los rezagados) y el Foro de Metacognición; el sábado para la Retroalimentación general a través de una videoconferencia y completar la Metacognición quienes no lo lograron el viernes, mientras que el domingo se dedica a la evaluación y publicación de notas por parte del docente.

Tabla 1. Créditos y hora académica

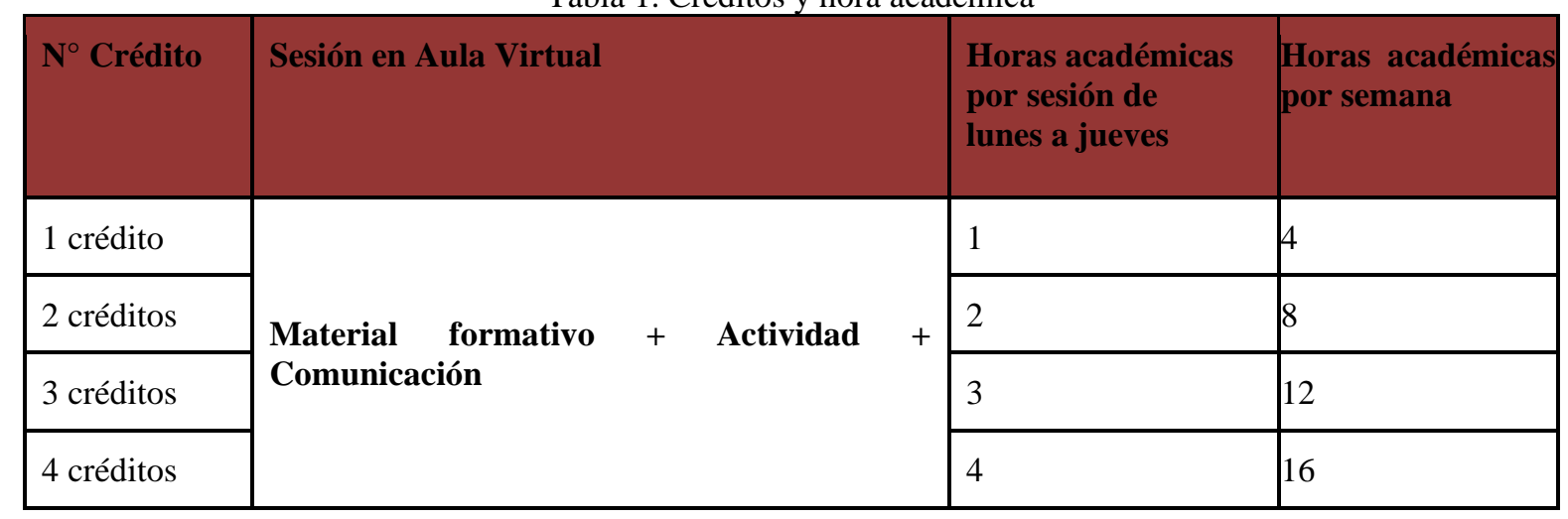

Tabla 2. Material formativo, actividades y comunicación por hora académica

\begin{tabular}{|c|c|c|}
\hline Tipo & Características & Tiempo \\
\hline Material formativo & Lectura, análisis y comprensión & Minutos \\
\hline $\begin{array}{l}\text { Material escrito (lectura, } \\
\text { separata, artículo, etc.) }\end{array}$ & $\begin{array}{l}10 \text { hojas } \\
\text { Tamaño de letra: } 12 \\
\text { Tipo de letra: Arial } \\
\text { Espaciado: } 11 / 2\end{array}$ & $45 \mathrm{~m}$ \\
\hline PPT textual & $\begin{array}{l}15 \text { diapositivas } \\
\text { Tamaño de letra: } 14 / 16 \\
\text { Tipo de letra: Arial } \\
\text { Espaciado: } 11 / 2\end{array}$ & $45 \mathrm{~m}$ \\
\hline PPT esquematizada & $\begin{array}{l}10 \text { diapositivas } \\
\text { Tamaño de letra: 14/16 } \\
\text { Tipo de letra: Arial } \\
\text { Espaciado: } 11 / 2\end{array}$ & $45 \mathrm{~m}$ \\
\hline Video & Videos máximo de 10 minutos & $45 \mathrm{~m}$ \\
\hline \multicolumn{3}{|l|}{ Actividades evaluadas } \\
\hline Tarea básica & Resolución de actividades & $45 \mathrm{~m}$ \\
\hline Tarea intermedia & Trabajos de investigación & $90 \mathrm{~m}$ \\
\hline Tarea compleja & $\begin{array}{l}\text { Elaboración y presentación de un producto } \\
\text { (blog, wiki, página web, etc.) }\end{array}$ & $135 \mathrm{~m}$ \\
\hline $\begin{array}{l}\text { Prácticas / Exámenes - } \\
\text { Preguntas cerradas }\end{array}$ & 20 preguntas & $60 \mathrm{~m}$ \\
\hline
\end{tabular}




\begin{tabular}{|c|c|c|}
\hline $\begin{array}{l}\text { Prácticas / Exámenes - } \\
\text { Preguntas mixtas }\end{array}$ & 10 preguntas & $60 \mathrm{~m}$ \\
\hline $\begin{array}{l}\text { Prácticas / Exámenes - } \\
\text { Preguntas abiertas }\end{array}$ & 4 preguntas & $60 \mathrm{~m}$ \\
\hline Foro temático /debate & $\begin{array}{l}\text { Debate de } 10 \text { líneas y réplica a dos } \\
\text { compañeros }\end{array}$ & $90 \mathrm{~m}$ \\
\hline Videoconferencia temática & $\begin{array}{l}\text { Presentación de un tema y } 6 \text { participaciones } \\
\text { de alumnos }\end{array}$ & $90 \mathrm{~m}$ \\
\hline Chat temático & $\begin{array}{l}\text { Responden a } 6 \text { preguntas planteadas y } \\
\text { retroalimentación }\end{array}$ & $60 \mathrm{~m}$ \\
\hline \multicolumn{3}{|l|}{ Comunicación } \\
\hline Foro de consultas & 1 consulta y 2 respuestas a sus compañeros & $30 \mathrm{~m}$ \\
\hline Correo & 2 envío y 1 respuesta & $30 \mathrm{~m}$ \\
\hline $\begin{array}{l}\text { Videoconferencia de } \\
\text { consultas }\end{array}$ & Se absuelven 10 consultas & $90 \mathrm{~m}$ \\
\hline Chat de consultas & Se absuelven 10 consultas & $60 \mathrm{~m}$ \\
\hline $\begin{array}{l}\text { Videoconferencia } \\
\text { retroalimentación }\end{array}$ & $\begin{array}{l}\text { Retroalimentación de actividades a } 10 \\
\text { participantes }\end{array}$ & $90 \mathrm{~m}$ \\
\hline Foro de retroalimentación & $\begin{array}{l}\text { Retroalimentación de actividades de } 20 \\
\text { participantes }\end{array}$ & $180 \mathrm{~m}$ \\
\hline
\end{tabular}

Nota: La metodología considera una hora académica de 45 minutos.

Tabla 3. Actividades complementarias

\begin{tabular}{|l|l|l|}
\hline \multirow{2}{*}{ Días } & Actividades & Tipos \\
\hline & Entregar actividades pendientes (Rezagados) & $\begin{array}{l}\text { Tareas } \\
\text { Prácticas } \\
\text { Exámenes }\end{array}$ \\
\cline { 2 - 4 } & Auto-reflexión /Metacognición & Foro \\
\hline \multirow{2}{*}{ Sábado } & Retroalimentación semanal del módulo & $\begin{array}{l}\text { Foro } \\
\text { Videoconferencia }\end{array}$ \\
\cline { 2 - 4 } & Auto-reflexión /Metacognición & Foro \\
\hline \multirow{2}{*}{ Domingo } & Promediar y publicar notas de módulo & Promedio de módulo \\
\hline
\end{tabular}

Un curso completo está formado por cuatro módulos que se desarrollan en un mes. Aquí podemos apreciar a través de un esquema el desarrollo de un módulo de un curso virtual de un programa de pregrado o posgrado en la USMP, este se desarrolla en una semana (lunes a domingo). 
Figura 3. Metodología para sesiones de clase virtual en la USMP

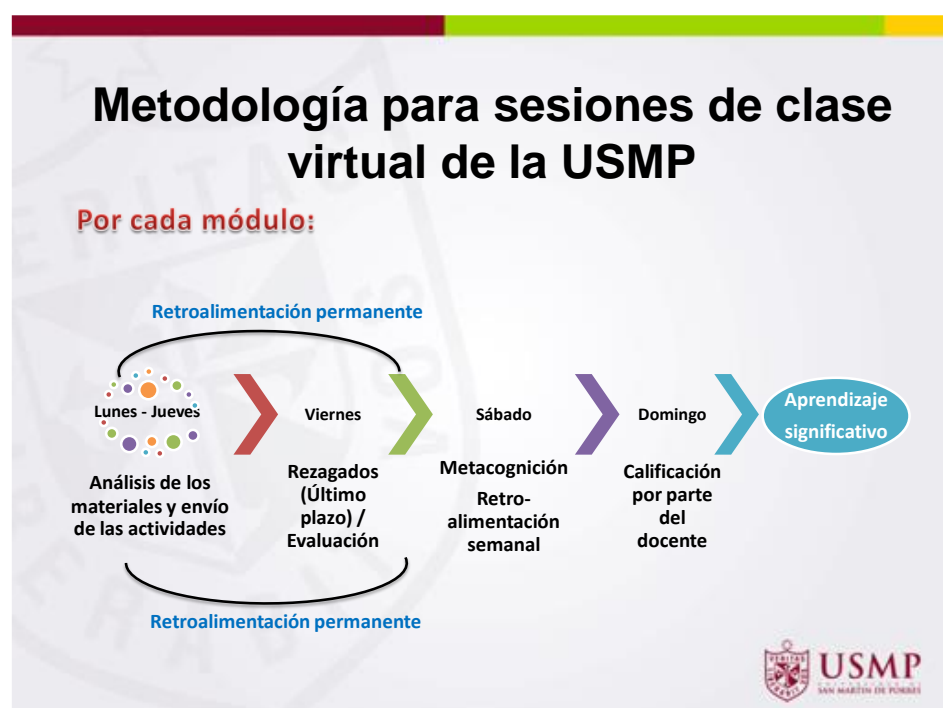

\section{DISEÑO PEDAGÓGICO DEL AULA VIRTUAL DE LA USMP}

La "Metodología para una sesión de un curso virtual", es la base para desarrollar con éxito un proceso de elaboración de materiales y el de un curso virtual de calidad en la plataforma Canvas, todo ello unificado con un Diseño Pedagógico del Aula Virtual, propio de la USMP Virtual, que estructura el aula virtual pedagógicamente en cuatros dimensiones (Flores, J. \& Huamán, M. 2013):

- Dimensión Informativa: esta dimensión presenta una información básica para situar a los estudiantes en su curso, con las pautas y recomendaciones necesarias, que les brinden una visión general de la asignatura que inician. Contiene: programación, temario, cronogramas, normas, etc.

- Dimensión Formativa: esta dimensión está formada por los recursos del curso, los cuales deben ser de conocimiento de los estudiantes para su formación. Presentan: contenido formativo; recursos o materiales de distintos formatos; como documentos textuales, hipertextos, presentaciones multimedia, esquemas, organizadores visuales, entre otros.

- Dimensión Experiencial: ofrece una propuesta de actividades variada, para que sean desarrolladas por los propios estudiantes, de tal manera que ellos logren experiencias de aprendizaje significativo en torno a dichos contenidos.

- Dimensión Comunicativa: en esta dimensión se desarrollan procesos comunicativos variados, fluidos y constantes entre el docente y sus estudiantes, así como entre los propios estudiantes a lo largo del tiempo de duración del curso (chat, foros, videoconferencias, etc.). 
Figura 4: Dimensiones del aula virtual USMP

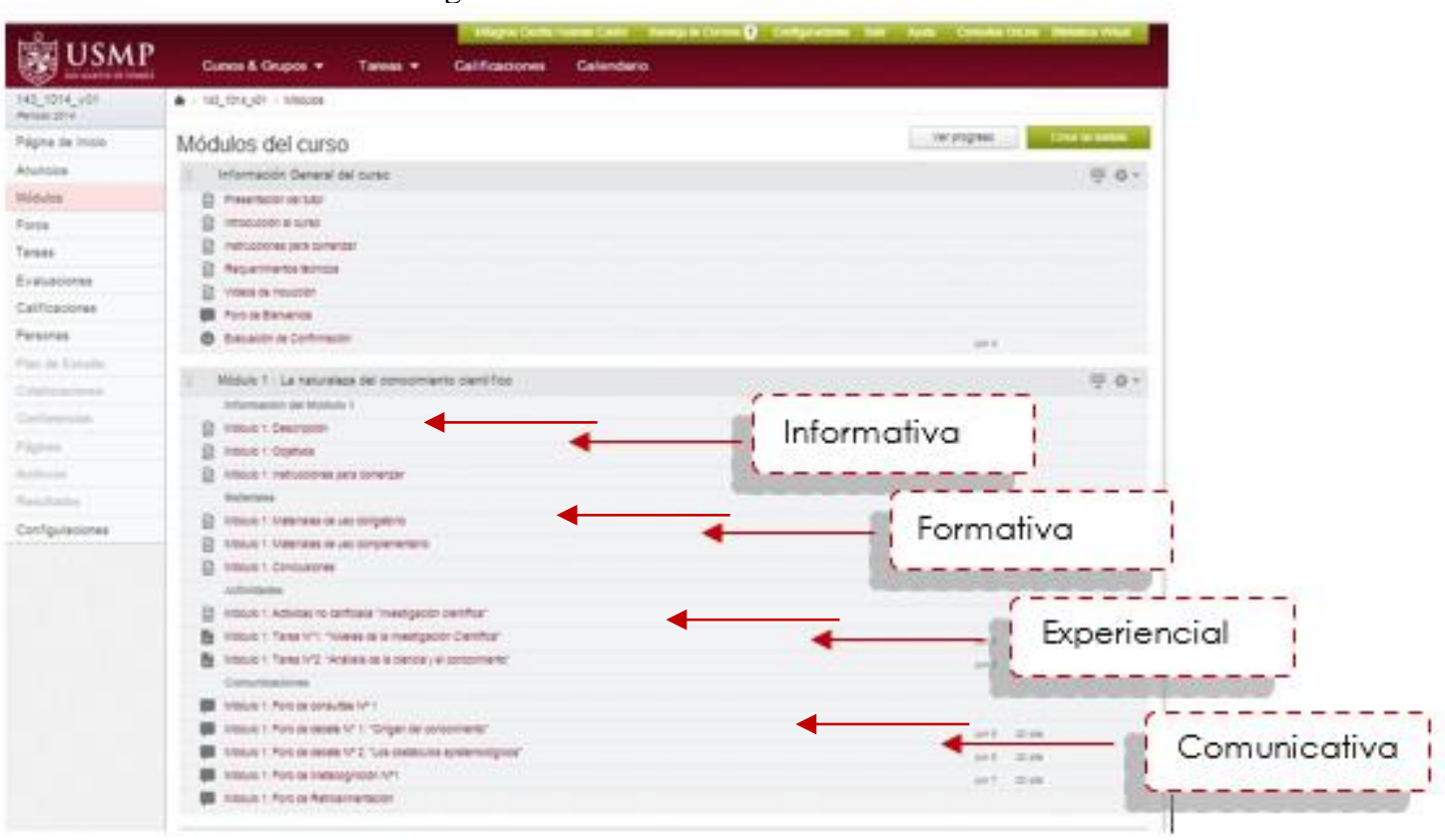

"La construcción de modelos pedagógicos que orienten el diseño y metodología de cursos virtuales, requiere el dominio de teorías tanto psicológicas como pedagógicas sobre el aprendizaje, así como de un amplio conocimiento de la población universitaria y de su contexto sociocultural”. Peña y Avendaño (2006). Esta premisa muestra la necesidad que comporta el hecho de implementar un curso virtual tomando en consideración los demás aspectos que entran en juego, que no son específicamente ni educativos ni tecnológicos, pero que también intervienen en el desenvolvimiento y producto de este recurso pedagógico. Así, los elementos sociales, culturales, psicológicos, lingüísticos, etc., generan un marco de comportamiento en cualquier comunidad, lo que afecta el diseño a emplear el aula virtual.

Para el diseño pedagógico del aula virtual nos basamos en investigaciones de la Universidad de La Laguna (España), difundidas en convenio con la Universidad de Salamanca. En base a esta investigación desarrollamos un diseño pedagógico para el aula virtual basado en las cuatro dimensiones que consideramos más relevantes para nuestro contexto. En consecuencia, nuestros cursos virtuales se caracterizan por incorporar en el diseño del aula virtual cuatro dimensiones claves para asegurar el proceso de enseñanza-aprendizaje basado en el E-Learning.

De esta manera se integran la "Metodología para sesiones de clase virtual” y el Diseño pedagógico del aula virtual" y dan como resultado nuestro "Modelo pedagógico de la USMP Virtual", que basado en estándares de calidad internacional e investigaciones, permiten desarrollar con éxito los cursos de programas de pregrado y posgrado virtual. 
Figura 5. Modelo Pedagógico de la USMP Virtual

Modelo Pedagógico de la USMP Virtual

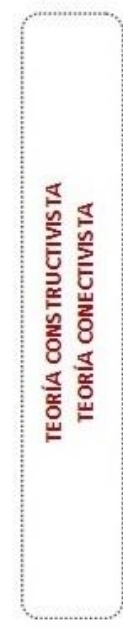

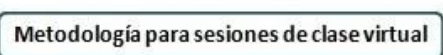
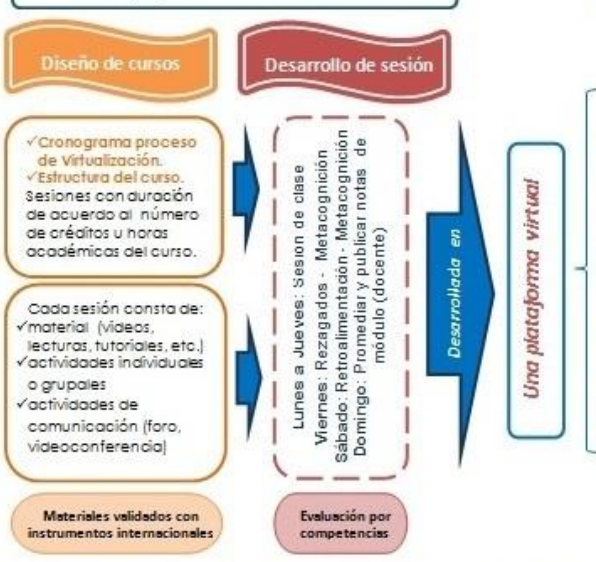

Aprendizaje activo
Diseño pedagógico del Aula Virtual

Dimensión informativa (Información)
- Presentación del curso, presentación del tutor - Presentación del curso, presentación del tutor,
sílabo, cronograma de actividades, guías y reglamentos.

Dimensión formativa (Materiales)

- Videos, lecturas, enlaces, presentaciones, etc.

Dimensión experiencial (Actividades)

- Actividades de aprendizaje individuales y

grupales, evaluaciones.

Dimensión comunicativa (Comunicación)

- Foros, chat, videoconferencias.

\section{CONCLUSIONES}

- La USMP cuenta con modelo pedagógico para cursos virtuales, basado en dos pilares; la metodología de cursos virtuales por créditos y horas académicas, y el diseño pedagógico del aula virtual.

- Los programas virtuales de la USMP se basan en el fundamento pedagógico de dos corrientes contemporáneas que lo respaldan, que son el constructivismo y el conectivismo, las cuales nos llevan al logro de un aprendizaje significativo en el entorno virtual.

- Nuestro modelo pedagógico cuenta con una metodología para sesiones de clase virtual por créditos y horas académicas, la cual se da en dos fases; diseño de cursos o programas virtuales, que cuenta con tres etapas; y el desarrollo de la sesión de clase virtual por crédito hora, que se da por módulos de una semana.

- La certificación de calidad de nuestros cursos pasa por dos niveles de revisión y validación; primero calidad de la USMP y luego, revisión de Academic PartnerShips bajo los estándares internacionales de calidad de Quality Matters (QM) y Quality Scorecard (QSC).

- Nuestro modelo pedagógico USMP se complementa con un diseño pedagógico del aula virtual, estructurado en cuatro dimensiones; informativa, formativa, experiencial y comunicativa. 


\section{REFERENCIAS}

Abbott, B. (1999). Presuppositions as nonassertions. Paper presented at the 73rd Annual.Meeting of the LSA, Los Angeles, CA. Recuperado de http://www.cca.org.mx/dds/cursos/cep21/modulo_1/main0_35.htm

Área, M., San Nicolás S. y Fariña, E. (2010). Buenas prácticas de aulas virtuales en la docencia universitaria presencial. España. Universidad de la Laguna.

Área, M. (2008). Evaluación del Campus Virtual de la Universidad de La Laguna. España. Laboratorio de Educación y Nuevas Tecnologías de la Laguna.

Ausubel, D. (2002). Adquisición y retención del conocimiento. Una perspectiva cognitiva. España: Paidós.

Barbera, E. (2001). La incógnita de la educación a distancia. Barcelona: ICE UB - Horsori.

Coll, C. (1991). Aprendizaje escolar y construcción del conocimiento. Buenos Aires: Ediciones Paidós.

Bruner, J. (1990). Acts of Meaning. Cambridge, MA: Harvard University Press.

Cabero, J. (2006). Propuestas de colaboración en educación a distancia y tecnologías para el aprendizaje. España: Universidad de Sevilla.

Esquerre, J. \& Huamán, M. (2021). Aplicación de videotutoriales para la mejora de las competencias matemáticas en los estudiantes de pregrado de la Universidad de San Martín de Porres. Vol. 2 No. 2 (2021): South Florida Journal of Development, Miami, v. 2, n. 2, apr./jun. 2021 Recuperado de: https://southfloridapublishing.com/ojs/index.php/jdev/article/view/216

Flores, J. \& Huamán, M. (2013). La USMP Virtual modelo de organización innovadora en e-learning. La educación a distancia en el Perú. VirtualEduca - ULADECH. Repositorio de la UNAM. México. Recuperado de: https://reposital.cuaieed.unam.mx:8443/xmlui/handle/20.500.12579/4106

Gallegos, S. (2006).Comunicación Familiar: un mundo de construcciones simbólicas y relacionales. (1 ed.) Manizales, Colombia: Ediciones Universidad de Chile.

Gros, B. (1997). Diseño y programas educativos. Pautas pedagógicas para la elaboración de software. Barcelona: Editorial Ariel.

Hacker, D. \& Keener, M. C. (1998). Metacognition in education: A focus on calibration. In J. Dunlosky, \& R. Bjork (Eds.), Handbook of Memory and Metacognition. Mahwah, NJ: Lawrence Erlbaum Associates.

Huamán, M. (2014). Propuesta académica para certificar un MOOC basada en una metodología elearning propia en la USMP Virtual. Revista EduTicInnova. Repositorio Digital de la UNAM. México. Recuperado de: https://reposital.cuaieed.unam.mx:8443/xmlui/handle/20.500.12579/3810

Jurado, R. (2010). Formamos el capital intelectual para la sociedad del conocimiento. Cuaderno 3: El diseño instruccional. Bogotá: Universidad Virtual. 
Klenowski,V (2005) Desarrollo de portafolios para el aprendizaje y la evaluación.(2 ed.). Madrid, España: Ediciones Narcea.

Méndez, A. (2002); Constructivismo social. Artículo curso 1 de Epistemología, Centros Comunitarios de Aprendizaje de Universidad Virtual del Tecnológico de Monterrey, México. Recuperado de http://www.cca.org.mx/dds/cursos/cep21/modulo_1/main0_35.htm

Moreira, M. (2012). La Teoría del Aprendizaje Significativo Crítico: un referente para organizar la enseñanza contemporánea. Recuperado http://www.fisem.org/www/union/revistas/2012/31/archivo 5 de volumen 31.pdf

Peña, M. \& Avendaño, B. (2006). Evaluación de la implementación del aula virtual en una institución de educación superior. Bogotá: Fundación Universitaria Konrad Lorenz.

Romero, A. \& Piedra, N. (2011). Calidad de contenidos en OCW. Recuperado de: http://reposital.cuaed.unam.mx:8080/jspui/bitstream/123456789/2706/1/romero_piedra_calidad_de contenidos_ocw.pdf

Sánchez, M. (2009). Mapas conceptuales como estrategia de aprendizaje con el Cmap Tools. (Tesis de maestría). México: Universidad Autónoma de Yucatán, Yucatán.

Siemens, G. (2004). Una teoría de aprendizaje para la era digital. Traducción de Diego Leal (2007). Colombia: Edu.Co.Blog.

Vargas, E. (2006); Constructivismo y modernización del aprendizaje. México: Enciclopedia de la Psicopedagogía.

Vygotski, L. (1979). El desarrollo de los procesos psicológicos superiores. Barcelona: Crítica. 